\title{
Rede Brasileira de Atenção e Estudos na Co-infecção Trypanosoma cruzi/HIV e em outras condições de imunossupressão
}

\author{
Brazilian Network of Attention and Studies on Trypanosoma cruzi/HIV \\ Co-infection and others immunossupression conditions
}

\author{
Eros Antonio de Almeida ${ }^{1}$, Alberto Novaes Ramos Junior ${ }^{3}$, \\ Dalmo Correia $^{2}$ e Maria Aparecida Shikanai-Yasuda ${ }^{4}$
}

A Rede Brasileira de Atenção e Estudos em Co-infecção Trypanosoma cruzi/HIV (e outras condições de imunossupressão) foi constituída em 2006, como estratégia de resposta organizada do Brasil aos novos desafios para o Sistema Único de Saúde (SUS)5. Tal entidade de indiscutível relevância, tanto para a saúde dos indivíduos acometidos quanto para a saúde pública, apresenta marcante morbi-mortalidade, requerendo não apenas estratégias de educação permanente para o manejo clínico, mas também para a vigilância epidemiológica ${ }^{10}$.

Desde a revisão da definição de caso de AIDS para a vigilância epidemiológica realizada em 1992, pelo Ministério da Saúde do Brasil, já havia o indicativo a partir das evidências na literatura científica nacional e internacional, de que a doença de Chagas poderia ter comportamento oportunista em indivíduos infectados por HIV em fase avançada ${ }^{3410}$. Em 2004, a reativação da doença de Chagas tornou-se um evento clínico de interesse epidemiológico como condição indicativa de AIDS $^{3}$ fato respaldado posteriormente em 2005 em reunião na Organização Pan-Americana da Saúde em Washington ${ }^{10}$.

De fato, a reativação da doença de Chagas em pacientes com co-infecção por HIV representa um evento potencialmente grave, que cursa com $100 \%$ de letalidade nos casos que recebem tratamento por até 30 dias, em contraste com os que o recebem por mais de 30 dias, quando a sobrevida é de aproximadamente $80 \%$. Em geral, em $2 / 3$ dos casos ou mais, o Sistema Nervoso Central é o principal sítio acometido, seguido por comprometimento miocárdico e por associação de ambos. Em contraste, em estudos prospectivos podem ser detectadas formas de pequena gravidade, semelhantes à síndrome da mononucleose infecciosa ou assintomáticas, que podem ser precocemente tratadas, resultando em sucesso terapêutico $0^{11011}$.

Adicionalmente, estudos prospectivos sobre a parasitemia em pacientes co-infectados têm mostrado que uma percentagem

1. Universidade Estadual de Campinas, Campinas, SP. 2. Universidade Federal do Triângulo Mineiro, Uberaba, MG. 3. Universidade Federal do Ceará, Fortaleza, CE. 4. Universidade de São Paulo, São Paulo, SP.

Endereço para correspondência: Dr. Eros Antonio de Almeida. Dept ${ }^{\circ}$ de Clínica Médica/FCM/UNICAMP. Rua Alexander Fleming 40, Cidade Universitária Zeferino Vaz, 13083-970 Campinas, São Paulo, SP.

Tel: 55 19 3521-7803/55; 19 9257-9269; Fax: 55 193289-4107.

e-mail: eros@fcm.unicamp.br

Recebido para publicação em 07/08/2009

Aceito em 28/08/2009 destes apresenta parasitemia bastante elevada ${ }^{2} 1011$. Foi possível estabelecer como limiar de reatividade pelo exame de xenodiagnóstico semi-quantitativo in vitro (com leitura individual das ninfas) o valor de $20 \%$, acima dos quais $50 \%$ dos pacientes co-infectados apresentam reativação da doença de Chagas ${ }^{1011}$.

No entanto, não se tem conhecimento da real prevalência desta co-infecção, seja no Brasil seja em outros países endêmicos, bem como da real frequiência de reativação, esta última estimada em 20\%. A magnitude da AIDS e da doença de Chagas no Brasil como condições crônicas ampliam a possibilidade de ocorrência deste evento ${ }^{310}$.

Um marco importante para a conformação das bases para constituição da rede foi a reunião realizada em 2000, em São Luís do Maranhão, durante o Congresso da Sociedade Brasileira de Medicina Tropical. 0 evento foi coordenado pela Dra. Vanize Macedo e contou com a participação de um grupo de especialistas que elaborou recomendações relativas à co-infecção Trypanosoma cruzi e HIV.

Com a conformação da rede durante a XXII Reunião Anual de Pesquisa Aplicada em Doença de Chagas e Leishmanioses, em Uberaba em outubro de 2006, seis reuniões técnico-científicas formais já foram realizadas 456789 . Estas reuniões tiveram a participação de profissionais de saúde e pesquisadores de referência no Brasil e em outros países na área de doença de Chagas e infecção por HIV/AIDS, bem como por representantes do Ministério da Saúde de diferentes áreas técnicas.

A proposta central para conformação da rede era de se ter um caráter amplo, não apenas englobando aspectos de organização de rede de serviços de saúde, mas também a necessidade de integração em rede a grupos internacionais já constituídos no tema, com estímulo a formação de novos grupos dentro de uma rede internacional ${ }^{56}$. Além de potencializar a atenção para a doença de Chagas, seria um espaço privilegiado para 0 desenvolvimento de estudos estratégicos na área ${ }^{67}$.

Uma série de missões estratégicas foi definida para a rede, incluindo o papel indutor para: estruturação da rede de atenção integral a pessoas com a doença de Chagas, em suas diferentes formas, em associação a estados de imunossupressão; desenvolvimento das ações dessa rede por meio de evidências obtidas a partir de pesquisas científicas, conduzidas segundo os preceitos éticos, incluindo aspectos clínicos, epidemiológicos, imunológicos e laboratoriais; estruturação de uma rede de 
educação permanente de profissionais da área de saúde para o manejo da doença de Chagas, em suas diferentes formas, em associação a estados de imunossupressão e identificação de temas prioritários a pesquisas no Brasil sobre a temática.

Desde a sua constituição até março de $2007^{45}$, a condução da rede esteve sob a responsabilidade provisória da Dra. Maria Aparecida Shikanai-Yasuda, da Universidade de São Paulo-SP. Em março de 2007, foram eleitos, em caráter formal, a Dra. Maria Aparecida Shikanai-Yasuda e Dr. Dalmo Correia Filho, da Universidade do Triângulo Mineiro-MG, respectivamente, para a coordenação e vice-coordenação da rede brasileira ${ }^{5}$. Em 2009, nova eleição foi realizada durante a sexta e última reunião da rede, em Recife, Pernambuco, durante o XLV Congresso da Sociedade Brasileira de Medicina Tropical. Foram eleitos o Dr. Eros Antonio de Almeida, da Universidade Estadual de Campinas-SP, como coordenador e o Dr. Dalmo Correia Filho como vice-coordenador da rede (recondução) ${ }^{9}$. Importante ressaltar que para a viabilização dos encontros e das atividades desta importante iniciativa, a rede vem contando desde a sua constituição com o apoio decisivo do Ministério da Saúde, especialmente da Secretaria de Vigilância em Saúde ${ }^{46789}$.

Dentro das atividades da rede, estiveram envolvidos profissionais da saúde e pesquisadores de referência na área no país, com o intuito de sistematizar as informações disponíveis sobre o tema e com vistas à estruturação de protocolos clínicos baseados em evidências. Foram testados instrumentos padronizados, que tem caráter de apoio a dados clínicos e laboratoriais em prontuários, optando-se por um instrumento simplificado com informações-chaves voltadas para a vigilância epidemiológica deste evento clínico ${ }^{789}$.

Em sua composição, a rede brasileira inclui centros de referência para pacientes com co-infecção por Trypanosoma cruzi e HIV, sendo apoiada pelos Programas Nacionais da Secretaria de Vigilância em Saúde, do Ministério da Saúde do Brasil, pelo Programa Nacional de Doença de Chagas (PNDCh), pelo Departamento Nacional de Controle de DST/AIDS e pela Rede Nacional de Laboratórios de Saúde Pública do país9.

Os centros de referência colaboram de forma direta na identificação de pacientes com co-infecção, inicialmente solicitando testes sorológicos para a triagem para infecção por HIV e para doença de Chagas, respectivamente. A caracterização da forma clínica é a etapa seguinte, que pode ser realizada nos serviços de referência iniciais ou em centros de referência de maior complexidade em função dos exames necessários. Para a avaliação quantitativa da parasitemia, exames parasitológicos indiretos ou moleculares poderão ser realizados em centros de maior complexidade ${ }^{78}$.

A proposta para identificação e mobilização da rede de serviços de saúde para composição da rede nacional definiu-se como critérios: atender casos de doença de Chagas; atender casos de co-infecção Trypanosoma cruzi e HIV; atender casos de reativação da doença de Chagas (por aids ou outras formas de imunossupressão); atender casos com infecção pelo HIV e/ou aids, com proximidade com os serviços de manejo da doença de Chagas $^{789}$.
Até 2008, segundo dados do Ministério da Saúde, estavam cadastrados no Brasil 37 centros de referência na Rede Nacional de Atenção e Estudos em Co-Infecção Trypanosoma cruzi/HIV, na sua maioria centros de referência para manejo da infecção por HIV e/ou para doença de Chagas. Mais de 130 casos de coinfecção Trypanosoma cruzi/ HIV foram cadastrados. Destes, aproximadamente, 20 apresentaram diagnóstico de reativação da doença de Chagas (casos registrados pelos centros do Hospital das Clínicas da Faculdade de Medicina da Universidade de São Paulo (FMUSP), da Universidade Federal do Triângulo Mineiro (UFTM), da Universidade Estadual de Campinas (UNICAMP) e da Universidade de Brasília) ${ }^{9}$. A partir da consolidação destes dados, e da literatura disponível, estima-se que para cada caso de reativação existam aproximadamente 2,6 casos de co-infecção.

Por fim, em relação às linhas de pesquisa potenciais e prioritárias, foram definidas pela rede: estudos epidemiológicos para definição da prevalência da co-infecção Trypanosoma cruzi/HIV de 1980 a 2006 (estudos longitudinais prospectivos e retrospectivos, em serviços de assistência a adultos/crianças/ gestantes ou de triagem de candidatos à doação de sangue), estudos clínico-epidemiológicos para avaliação da incidência e de fatores preditores da reativação bem como para definição de protocolo para seguimento; estudos clínico-epidemiológicos para avaliação de novas modalidades terapêuticas e profiláticas bem como para estimativa de recidivas em pacientes tratados em remissão parasitológica; estudos sobre as características genotípicas das subpopulações do parasito na reativação bem como sobre a influência de subtipos HIV-1 e HIV-2 na reativação e a interação com diferentes subpopulações de Trypanosoma cruzi e, finalmente, estudos sobre a fisiopatogenia e a imunopatogenia da reativação, incluindo estudos para validação de técnicas para a quantificação da parasitemia como fator prognóstico de reativação ${ }^{6780}$.

A proposta é, em um futuro próximo, ampliar o número de serviços envolvidos na rede e articular a rede brasileira a outras redes em diferentes países da América Latina e de outros continentes que se debruçam na orientação da assistência e na pesquisa da doença de Chagas na infecção por HIV e em outras formas de imunossupressão. De uma forma geral, espera-se ampliar os olhares para a doença de Chagas como doença negligenciada.

\section{Centros participantes:}

Ademir Rocha (in memorian) - Universidade Federal de Uberlândia, Minas Gerais

Alberto Novaes Ramos Jr - Universidade Federal do Ceará, Ceará

Alejandro Ostermyer Luquetti - Universidade Federal de Goiás, Goiás

Alejandro Marcel Hasslocher Moreno - Instituto de Pesquisa Clínica Evandro Chagas, FIOCRUZ, Rio de Janeiro

Aloísio Falqueto - Universidade Federal do Espírito Santo, Espírito Santo 
Ana Marli Sartori - Universidade de São Paulo, São Paulo Ana Nilce Maia Elkhoury - SVS/Ministério da Saúde, Distrito Federal

Ana Yecê das Neves Pinto - Instituto Evandro Chagas, Pará

Ângela Maria da Silva - Universidade Federal de Sergipe, Sergipe

Artur Maia Paz - Universidade Federal de Alagoas, Alagoas

Breno Riegel dos Santos - Hospital Nossa Senhora da Conceição, Rio Grande do Sul

Carlos Henrique Nery Costa - Universidade Federal do Piauí, Piauí

Claudia Mendonça Bezerra - Secretaria Estadual de Saúde do Ceará, Ceará

Cleudson Nery Castro - Universidade de Brasília, Distrito Federal

Clovis Arns da Cunha - Universidade Federal do Paraná, Paraná

Dalmo Correia Filho - Universidade Federal do Triângulo Mineiro, Minas Gerais

Denise Lima Mascarenhas - Hospital Regional Cleriston Andrade de Feira de Santana, Bahia

Dirceu Greco - Universidade Federal de Minas Gerais, Minas Gerais

Edna Moliene de Souza - Instituto Adolpho Lutz, São Paulo

Elenild de Goes Costa - Secretaria Estadual de Saúde do Pará, Pará

Eliana Furtado Moreira - Fundação Ezequiel Dias, Minas Gerais

Emanuel Costa Martins - SVS/PNCDCh/Ministério da Saúde, Distrito Federal

Erica Tatto - Secretaria Municipal de Saúde de Caxias do Sul, Rio Grande do Sul

Eros Antonio de Almeida - Universidade Estadual de Campinas, São Paulo

Fernando Góngora Rubio - Universidade Estadual de São José do Rio Preto, São Paulo

Fernando Pedrosa - Universidade Federal de Alagoas, Alagoas

Jaime Costa da Silva - SVS/PNCDCh/Ministério da Saúde, Distrito Federal

Jorge Guerra - Fundação de Medicina Tropical, Amazonas

Jorim Severino Ithamar - Hospital Presidente Vargas, Maranhão

José Fernando de Castro Figueiredo - Universidade de São Paulo - Ribeirão Preto, São Paulo

Karim Yaqub Ibrahim - Universidade de São Paulo, São Paulo

Lourival Rodrigues Marsola - Universidade Federal do Pará, Pará

Luciano Correia Ribeiro - Universidade Federal de Mato Grosso, Mato Grosso
Marcelo Simão Ferreira - Universidade Federal de Uberlândia, Minas Gerais

Márcia Hueb - Universidade Federal de Mato Grosso, Mato Grosso

Margareth Dayse Medeiros Monteiro - Universidade Estadual de Ciências da Saúde de Alagoas, Alagoas

Margareth Dioz Silva - Universidade Federal de Mato Grosso, Mato Grosso

Maria Aparecida Shikanai-Yasuda - Universidade de São Paulo, São Paulo

Marília Maria dos Santos Severo - Universidade Federal de Ciências da Saúde de Porto Alegre, Rio Grande do Sul

Mário Steindel - Universidade Federal de Santa Catarina, Santa Catarina

Maurício Borborema de Medeiros - Fundação de Medicina Tropical, Amazonas

Nancy Silva - Hospital Geral Roberto Santos, Bahia

Noêmia Barbosa Carvalho - Universidade de São Paulo, São Paulo

Otaciano de Paula Campos - Hospital Santo Amaro, Bahia

Renee de Oliveira Nascimento - Serviço de Ambulatório Especializado em DST/HIV/AIDS-Salgadinho, Alagoas

Roberto da Justa Pires Neto - Universidade Federal do Ceará, Ceará

Roberto José da Silva Badaró - Universidade Federal da Bahia, Bahia

Ruth Moreira Leite - Secretaria Estadual de Saúde de São Paulo, São Paulo

Silene Maria Fontes Barreto Dantas - Hospital Couto Maia, Bahia

Simone Monzani Vivaldini - CGLAB/SVS/Ministério da Saúde, Distrito Federal

Soraya Oliveira dos Santos - SVS/PNCDCh/Ministério da Saúde, Distrito Federal

Suelene Mamede de Oliveira - HEMOBRAS/Ministério da Saúde, Distrito Federal

Suterlânio Teixeira Rocha - Centro de Referência em Doenças Endêmicas de Jequié, Bahia

Tânia Marcial - Hospital Eduardo de Menezes, Minas Gerais

Unaí Tupinambás - Universidade Federal de Minas Gerais, Minas Gerais

Vera da Costa Valente - Instituto Evandro Chagas, Pará

Vera Lúcia Carvalho da Silva - SVS/PNCDCh/Ministério da Saúde, Distrito Federal

\section{REFERÊNCIAS}

1. Cordova E, Boschi A, Ambrosioni J, Cudos C, Corti M. Reactivation of Chagas' disease with central nervous system involvement in HIV-infected patients in Argentina, 1992-2007. International Journal of Infectious Disease 12:587-592, 2008. 
2. Perez-Ramirez L, Barnabe C, Sartori AM, Ferreira MS, Tolezano JE, Nunes EV, Burgarelli MK, Silva AC, Shikanai-Yasuda MA, Lima JN, Da-Cruz AM, Oliveira OC, Guilherme C, Bastrenta B, Tibayrenc M. Clinical analysis and parasite genetic diversity in human immunodeficiency virus/Chagas' disease coinfections in Brazil. The American Journal of Tropical Medicine and Hygiene 61:198-206, 1999.

3. Ramos Jr AN. Inclusão da reativação da doença de Chagas como uma condição definidora de AIDS para fins de vigilância epidemiológica no Brasil. Revista da Sociedade Brasileira de Medicina Tropical 37:192-193, 2004.

4. Rede Brasileira de Atenção e Estudos em Co-infecção Trypanosoma cruzi/HIV. Relatório técnico $1^{\mathrm{a}}$ Reunião, 2006.

5. Rede Brasileira de Atenção e Estudos em Co-infecção Trypanosoma cruzi/HIV. Relatório técnico $2^{\mathrm{a}}$ Reunião, 2007.

6. Rede Brasileira de Atenção e Estudos em Co-infecção Trypanosoma cruzi/HIV. Relatório técnico $3^{\mathrm{a}}$ Reunião, 2007.
7. Rede Brasileira de Atenção e Estudos em Co-infecção Trypanosoma cruzi/HIV. Relatório técnico $4^{\mathrm{a}}$ Reunião, 2008.

8. Rede Brasileira de Atenção e Estudos em Co-infecção Trypanosoma cruzi/HIV. Relatório técnico $5^{\mathrm{a}}$ Reunião, 2008.

9. Rede Brasileira de Atenção e Estudos em Co-infecção Trypanosoma cruzi/HIV. Relatório técnico $6^{a}$ Reunião, 2009.

10. Rocha A, Ramos Jr AN, Sartori AMC, Correa D, Gontijo ED, Tatto E, Almeida E, Pereira GF, Silva JC, Figueredo JFC, Ferreira MS, Shikanai-Yasuda MA, Pontes RJS, Santos SOS. Recomendações para diagnóstico, tratamento e acompanhamento da co-infecção Trypanosoma cruzi - vírus da imunodeficiência humana. Revista da Sociedade Brasileira de Medicina Tropical 39:392-415, 2006

11. Sartori AM, Ibrahim KY, Nunes Westphalen EV, Braz LM, Oliveira OC Jr, Gakiya E, Lopes MH, Shikanai-Yasuda MA. Manifestations of Chagas' disease (American trypanosomiasis) in patients with HIV/AIDS. Annals of Tropical Medicine \& Parasitology 101:31-50, 2007. 